\title{
ON LINEAR EQUATIONS WITH POLYNOMIAL COEFFICIENTS
}

\author{
WOJCIECH KUCHARZ \\ Institute of Mathematics, Faculty of Mathematics and Computer Science \\ Jagiellonian University, Łojasiewicza 6, 30-348 Kraków, Poland \\ ORCID:0000-0001-7531-9495 E-mail: Wojciech.Kucharz@im.uj.edu.pl
}

\begin{abstract}
We give a short survey of result on continuous (resp. continuous semialgebraic or regulous) solutions of linear equations with polynomial coefficients.
\end{abstract}

1. The main problems. Unless explicitly stated otherwise, by a function we will mean a real-valued function.

Consider a linear equation

$$
f_{1} y_{1}+\ldots+f_{r} y_{r}=\varphi
$$

where $f_{1}, \ldots, f_{r}, \varphi$ are continuous functions on $\mathbb{R}^{n}$. Fefferman and Kollár [5] study the following two questions:

Question 1. Is there a continuous solution of 1.1 ? In other words, can one find a solution $y_{1}=\varphi_{1}, \ldots, y_{r}=\varphi_{r}$ of $(1.1)$, where the $\varphi_{i}$ are continuous functions on $\mathbb{R}^{n}$ ?

Question 2. Suppose that (1.1) has a continuous solution. If $\varphi$ and the $f_{i}$ have some regularity properties, can one find a continuous solution of 1.1 which has the same (or weaker) properties?

An algebraic version of Question 11 for complex-valued functions on $\mathbb{C}^{n}$, was posed by Brenner [3] and led him to the notion of continuous closure of ideals. Epstein and Hochster 44 presented a detailed discussion of the continuous closure and other closure operations on ideals. Kollár 6] gave a characterization of the continuous closure and extended this notion to sheaves.

2010 Mathematics Subject Classification: 26C15, 13A15, 14P99, 54C65.

Key words and phrases: continuous functions, semialgebraic functions, regulous functions, linear equations.

The paper is in final form and no version of it will be published elsewhere. 
It is fairly obvious that in general (1.1) has no continuous solution. Indeed, let

$$
\mathcal{H}\left(f_{1}, \ldots, f_{r} ; \varphi\right)=\left\{H\left(f_{1}, \ldots, f_{r} ; \varphi\right)_{x}\right\}_{x \in \mathbb{R}^{n}}
$$

where

$$
H\left(f_{1}, \ldots, f_{r} ; \varphi\right)_{x}:=\left\{\left(v_{1}, \ldots, v_{r}\right) \in \mathbb{R}^{r}: f_{1}(x) v_{1}+\ldots+f_{r}(x) v_{r}=\varphi(x)\right\} .
$$

If $H\left(f_{1}, \ldots, f_{r} ; \varphi\right)_{x}$ is the empty set for some $x \in \mathbb{R}^{n}$, then (1.1) cannot have a continuous solution.

Definition 3. We say that equation (1.1) satisfies the pointwise test if for every $p \in \mathbb{R}^{n}$ it has a solution $y_{1}=\varphi_{1}^{(p)}, \ldots, y_{r}=\varphi_{r}^{(p)}$, where the $\varphi_{i}^{(p)}$ are functions on $\mathbb{R}^{n}$ which are continuous at $p$.

Evidently, the pointwise test is a necessary condition for equation (1.1) to have a continuous solution. However, the following example, due to Hochster and discussed in [5. Example 3.4], shows that the pointwise test is not a sufficient condition in general.

EXAmple 4 . On $\mathbb{R}^{3}$ with coordinates $\left(x_{1}, x_{2}, x_{3}\right)$, the linear equation

$$
\left(x_{1}^{2}\right) y_{1}+\left(x_{2}^{2}\right) y_{2}+\left(x_{1} x_{2} x_{3}^{2}\right) y_{3}=x_{1} x_{2} x_{3}
$$

satisfies the pointwise test but it has no continuous solution.

In what follows, both 1.2 and the pointwise test will play an essential role.

2. Singular affine bundles. Fix positive integers $n$ and $r$. By convention, the empty set will be regarded as an affine subspace of $\mathbb{R}^{r}$.

Definition 5. A singular affine bundle (or bundle for short) is a family $\mathcal{H}=\left\{H_{x}\right\}_{x \in \mathbb{R}^{n}}$ of affine subspaces $H_{x} \subseteq \mathbb{R}^{r}$. The affine subspaces $H_{x}$ are the fibers of the bundle $\mathcal{H}$ (some fibers are allowed to be empty). A section of $\mathcal{H}$ is a continuous map $s: \mathbb{R}^{n} \rightarrow \mathbb{R}^{r}$ such that $s(x) \in H_{x}$ for all $x \in \mathbb{R}^{n}$.

An example of a bundle is provided by $\mathcal{H}\left(f_{1}, \ldots, f_{r} ; \varphi\right)$ in $(1.2)$. Clearly, equation (1.1) has a continuous solution if and only if the bundle $\mathcal{H}\left(f_{1}, \ldots, f_{r} ; \varphi\right)$ has a section.

Let $\mathcal{H}=\left\{H_{x}\right\}_{x \in \mathbb{R}^{n}}$ be a bundle. One readily checks that $\mathcal{H}^{\prime}=\left\{H_{x}^{\prime}\right\}_{x \in \mathbb{R}^{n}}$, where

$$
H_{x}^{\prime}:=\left\{v \in H_{x}: \operatorname{dist}\left(v, H_{y}\right) \rightarrow 0 \text { as } y \rightarrow x\right\},
$$

is a bundle. Actually, $\mathcal{H}^{\prime}$ is a subbundle of $\mathcal{H}$, that is, $H_{x}^{\prime} \subseteq H_{x}$ for all $x \in \mathbb{R}^{n}$. Furthermore, $\mathcal{H}$ and $\mathcal{H}^{\prime}$ have the same sections. In [5], $\mathcal{H}^{\prime}$ is called the Glaeser refinement of $\mathcal{H}$. Iterating the Glaeser refinement, we obtain a sequence of bundles $\mathcal{H}^{0}, \mathcal{H}^{1}, \mathcal{H}^{2}, \ldots$, where $\mathcal{H}^{0}=\mathcal{H}$ and $\mathcal{H}^{i+1}$ is the Glaeser refinement of $\mathcal{H}^{i}$ for each $i \geq 0$.

The following result is established in [5, Lemmas 5 and 6].

TheOREM 6. Let $\mathcal{H}=\left\{H_{x}\right\}_{x \in \mathbb{R}^{n}}$ be a bundle.

(i) $\mathcal{H}^{i}=\mathcal{H}^{2 r+1}$ for $i \geq 2 r+1$.

(ii) If $\mathcal{H}=\mathcal{H}^{\prime}$ and if each fiber $H_{x}$ is nonempty, then $\mathcal{H}$ has a section.

Hence, Theorem 6 provides the following answer to Question 1

COROLlary 7. Equation (1.1) has a continuous solution if and only if each fiber of the bundle $\mathcal{H}\left(f_{1}, \ldots, f_{r} ; \varphi\right)^{2 r+1}$ is nonempty. 
A lot more on Question 1 and the Glaeser refinement is contained in [5]. However, it is not possible to state these result in a concise form.

3. Continuous semialgebraic and regulous solutions. In what follows we focus on Question 2 The problem is quite hard even if $\varphi$ and the $f_{i}$ are polynomial functions.

Recall that a subset of $\mathbb{R}^{n}$ is semialgebraic if it is a finite Boolean combination of sets of the form

$$
\left\{x \in \mathbb{R}^{n}: p(x)=0\right\} \text { and }\left\{x \in \mathbb{R}^{n}: q(x)>0\right\},
$$

where $p$ and $q$ are polynomial functions on $\mathbb{R}^{n}$. A function $f: S \rightarrow \mathbb{R}$, defined on a semialgebraic set $S \subseteq \mathbb{R}^{n}$, is semialgebraic if its graph is a semialgebraic subset of $\mathbb{R}^{n} \times \mathbb{R}$.

The main result of [5] concerning Question 2 is a rather complicated algorithm of which some parts may not be effectively doable. Nevertheless, the general structure of the algorithm yields the following.

THEOREM 8. Assume that $f_{1}, \ldots, f_{r}$ are polynomial functions on $\mathbb{R}^{n}$ and $\varphi$ is a continuous semialgebraic function on $\mathbb{R}^{n}$. If the equation

$$
f_{1} y_{1}+\ldots+f_{r} y_{r}=\varphi
$$

has a continuous solution, then it also has a continuous semialgebraic solution.

A different approach to Question 2 is presented by Sokantika and Thamrongthanyalak [12. They construct definable selections of set-valued maps in the o-minimal setting and, in particular, obtain a more general version of Theorem 8

Henceforth, we consider Question 2 for functions of other types.

Definition 9. A function $f$ on $\mathbb{R}^{n}$ is said to be regulous if it is continuous and there exist two polynomial functions $p, q$ on $\mathbb{R}^{n}$ such that $q$ is not identically 0 and $f=p / q$ on the set $\left\{x \in \mathbb{R}^{n}: q(x) \neq 0\right\}$.

Regulous functions appear in a natural way in many different contexts; see [7, 8, 11] and the reference therein. They also play a role in Question 22 By [2, Proposition 2.2.2], regulous functions are semialgebraic.

EXAMPLE 10 . On $\mathbb{R}^{2}$ with coordinates $\left(x_{1}, x_{2}\right)$, the linear equation

$$
\left(x_{1}^{3}\right) y_{1}+\left(x_{2}^{3}\right) y_{1}=x_{1}^{2} x_{2}^{2}
$$

has no $\mathcal{C}^{\infty}$ solution. It has a regulous solution $\left(y_{1}, y_{2}\right)=\left(\varphi_{1}, \varphi_{2}\right)$, where

$$
\begin{aligned}
& \varphi_{1}\left(x_{1}, x_{2}\right)= \begin{cases}\frac{x_{1}^{5} x_{2}^{2}}{x_{1}^{6}+x_{2}^{6}} & \text { for }\left(x_{1}, x_{2}\right) \neq(0,0) \\
0 & \text { for }\left(x_{1}, x_{2}\right)=(0,0),\end{cases} \\
& \varphi_{2}\left(x_{1}, x_{2}\right)= \begin{cases}\frac{x_{1}^{2} x_{2}^{5}}{x_{1}^{6}+x_{2}^{6}} & \text { for }\left(x_{1}, x_{2}\right) \neq(0,0) \\
0 & \text { for }\left(x_{1}, x_{2}\right)=(0,0) .\end{cases}
\end{aligned}
$$

It can happen that equation (1.1), where $\varphi$ and $f_{i}$ are polynomial functions, has a continuous semialgebraic solution but has no regulous solution. 
EXAMPLE 11 . On $\mathbb{R}^{3}$ with coordinates $\left(x_{1}, x_{2}, x_{3}\right)$, the linear equation

$$
\left(x_{1}^{3} x_{2}\right) y_{1}+\left(x_{1}^{3}-\left(1+x_{3}^{2}\right) x_{2}^{3}\right) y_{2}=x_{1}^{4}
$$

has a continuous semialgebraic solution $\left(y_{1}, y_{2}\right)=\left(\varphi_{1}, \varphi_{2}\right)$, where

$$
\varphi_{2}\left(x_{1}, x_{2}, x_{3}\right)= \begin{cases}\frac{x_{1}^{3}\left(x_{1}, x_{2}, x_{3}\right)=\left(1+x_{3}^{2}\right)^{1 / 3}}{x_{1}^{2}+\left(1+x_{3}^{2}\right)^{1 / 3} x_{1} x_{2}+\left(1+x_{3}\right)^{2 / 3} x_{2}^{2}} & \text { for }\left(x_{1}, x_{2}\right) \neq(0,0) \\ 0 & \text { for }\left(x_{1}, x_{2}\right)=(0,0) .\end{cases}
$$

Now suppose that $\left(y_{1}, y_{2}\right)=\left(\psi_{1}, \psi_{2}\right)$ is a regulous solution of 3.1 . We obtain a contradiction as follows. The algebraic surface

$$
S:=\left\{\left(x_{1}, x_{2}, x_{3}\right) \in \mathbb{R}^{3}: x_{1}^{3}-\left(1+x_{3}^{2}\right) x_{2}^{3}=0\right\}
$$

is a real analytic submanifold of $\mathbb{R}^{3}$ since it can also be described by a real analytic equation

$$
x_{1}-\left(1+x_{3}^{2}\right)^{1 / 3} x_{2}=0 .
$$

Note that the $x_{3}$-axis is contained in $S$. Substituting $\left(y_{1}, y_{2}\right)=\left(\psi_{1}, \psi_{2}\right)$ into $\sqrt{3.1}$ and restricting to $S$, we obtain

$$
\left.\left(\left.x_{1}^{3} x_{2}\right|_{S}\right) \psi_{1}\right|_{S}=\left.x_{1}^{4}\right|_{S},
$$

hence

$$
\left.\psi_{1}\right|_{S \backslash\left(x_{3} \text {-axis }\right)}=\left.\frac{x_{1}}{x_{3}}\right|_{S \backslash\left(x_{3} \text {-axis }\right)}=\left.\left(1+x_{3}^{2}\right)^{1 / 3}\right|_{S \backslash\left(x_{3} \text {-axis }\right)} .
$$

Since $S \backslash\left(x_{3}\right.$-axis $)$ is dense in $S$, we get

$$
\left.\psi_{1}\right|_{S}=\left.\left(1+x_{3}^{2}\right)^{1 / 3}\right|_{S}
$$

and consequently

$$
\left.\psi_{1}\right|_{\left(x_{3} \text {-axis }\right)}=\left(1+x_{3}^{2}\right)^{1 / 3} .
$$

The last equality cannot hold, the function $\psi_{1}$ being regulous.

Example 11 comes from [8]. It is worthwhile to record the following generalization.

EXAMPLE 12. For each $n \geq 3$, one can interpret (3.1) as a linear equation on $\mathbb{R}^{n}$ with coordinates $\left(x_{1}, \ldots, x_{n}\right)$. Then (3.1) has a continuous semialgebraic solution on $\mathbb{R}^{n}$ but no regulous solution on $\mathbb{R}^{n}$.

The following question is of interest [8].

QUESTION 13. For which regulous functions $f_{1}, \ldots, f_{r}, \varphi$ on $\mathbb{R}^{n}$, the linear equation

$$
f_{1} y_{1}+\ldots+f_{r} y_{r}=\varphi
$$

has a regulous solution?

As of this writing, Question 13 remains very much open for $n \geq 3$. The case $n=1$ is an easy exercise. The case $n=2$ to which we turn next was settled by Kucharz and Kurdyka [10]. It is convenient to start with a result on Question 1 and the pointwise test. 
THEOREM 14. Let $f_{1}, \ldots, f_{r}$ be regulous functions and $\varphi$ a continuous function, all defined on $\mathbb{R}^{2}$. Then the linear equation

$$
f_{1} y_{1}+\ldots+f_{r} y_{r}=\varphi
$$

has a continuous solution if and only if it satisfies the pointwise test.

Theorem 14 shows that the phenomenon described in Example 4 can occur only for functions on $\mathbb{R}^{n}$ with $n \geq 3$.

THEOREM 15. Let $f_{1}, \ldots, f_{r}, \varphi$ be regulous functions on $\mathbb{R}^{2}$. If the linear equation

$$
f_{1} y_{1}+\ldots+f_{r} y_{r}=\varphi
$$

has a continuous solution, then it also has a regulous solution.

Combining Theorems 14 and 15 we obtain for functions on $\mathbb{R}^{2}$ a satisfactory partial answer to Question 2 and a complete answer to Question 13. In [10], Theorems 14 and 15 are stated and proved in a more general setting, namely for functions defined on a nonsingular real algebraic set of dimension 2 .

Let $f_{1}, \ldots, f_{r}, \varphi$ be polynomial functions on $\mathbb{R}^{n}$ and suppose that the linear equation

$$
f_{1} y_{1}+\ldots+f_{r} y_{r}=\varphi
$$

has a continuous solution. By Theorem 8 , there is also a continuous semialgebraic solution. Can one find a continuous semialgebraic solution which has some additional regularity properties? Several suggestions were put forward in [9] and in discussions among the experts. Recently, Adamus and Seyedinejad [1] provided counterexamples to some of them.

Acknowledgements. The author was partially supported by the National Science Center (Poland) under grant number 2014/15/B/ST1/00046.

\section{References}

[1] J. Adamus, H. Seyedinejad, On solutions of linear equations with polynomial coefficients, Ann. Polon. Math. 121 (2018), 1-6.

[2] J. Bochnak, M. Coste, M.-F. Roy, Real Algebraic Geometry, Ergeb. Math. Grenzgeb. (3) 36, Springer, Berlin, 1998.

[3] H. Brenner, Continuous solutions to algebraic forcing equations, arXiv:math/0608611v2

[4] N. Epstein, M. Hochster, Continuous closure, axes closure, and natural closure, Trans. Amer. Math. Soc. 370 (2018), 3315-3362.

[5] C. Fefferman, J. Kollár, Continuous solutions of linear equations, in: From Fourier Analysis and Number Theory to Radon Transforms and Geometry, Dev. Math. 28, Springer, New York, 2013, 233-282.

[6] J. Kollár, Continuous closure of sheaves, Michigan Math. J. 61 (2012), 475-491.

[7] J. Kollár, W. Kucharz, K. Kurdyka, Curve-rational functions, Math. Ann. 370 (2018), 39-69.

[8] J. Kollár, K. Nowak, Continuous rational functions on real and p-adic varieties, Math. Z. 279 (2015), 85-97.

[9] W. Kucharz, Nash regulous functions, Ann. Polon. Math. 119 (2017), 275-289. 
[10] W. Kucharz, K. Kurdyka, Linear equations on real algebraic surfaces, Manuscripta Math. 154 (2017), 285-296.

[11] W. Kucharz, K. Kurdyka, From continuous rational to regulous functions, in: Proceedings of the International Congress of Mathematicians-Rio de Janeiro 2018, Vol. 2, World Sci. Publ., Hackensack, NJ, 2019, 719-747.

[12] S. Sokantika, A. Thamrongthanyalak, Definable continuous selections of set-valued maps in o-minimal expansions of the real field, Bull. Pol. Acad. Sci. Math. 65 (2017), 97-105. 